\title{
Membrane Lipid Replacement: Clinical Studies Using a Natural Medicine Approach to Restoring Membrane Function and Improving Health
}

\author{
Garth L. Nicolson \\ Department of Molecular Pathology, The Institute for Molecular Medicine, Huntington Beach, CA, USA \\ Email: gnicolson@immed.org
}

Received 5 January 2016; accepted 17 February 2016; published 22 February 2016

Copyright (C) 2016 by author and Scientific Research Publishing Inc. This work is licensed under the Creative Commons Attribution International License (CC BY). http://creativecommons.org/licenses/by/4.0/

c) (7) Open Access

\begin{abstract}
Functional oral supplements containing cell membrane glycerolphospholipids and antioxidants have been used to safely replace damaged membrane lipids that accumulate during aging and in various clinical conditions. This approach differs from other dietary and intravenous interventions in the composition of phospholipids and the presence of fructooligosaccharides that protect the phospholipids against oxidation and bile and enzymatic damage. Various chronic clinical conditions are characterized by membrane phospholipid oxidative damage, resulting in loss of cellular function. Recent clinical trials have shown the benefits of Membrane Lipid Replacement in replenishing damaged membrane lipids and restoring mitochondrial function, resulting in reductions in fatigue in aged subjects and patients with a variety of clinical diagnoses. Recent in vitro experiments with nonphysiological concentrations of phospholipids did not result in enhancement of mitochondrial electron transport enzyme activities. This can be explained by the use of the wrong phospholipid fatty acids, over-dilution of membrane constituents and mitochondrial swelling. A similar phenomenon was seen when human sperm were incubated in vitro with high concentrations of glycerolphospholipids and their motility was assessed. Only lower, more physiological concentrations of glycerolphospholipids stimulated sperm motility. Additional studies are needed to determine the functional effects of Membrane Lipid Replacement on other cellular membranes, such as the plasma membrane and other intracellular membranes of various cells and tissues.
\end{abstract}

\section{Keywords}

Membrane Phospholipids, Lipid Oxidation, Mitochondrial Function, Fatigue, Clinical Trials, Membrane Lipid Replacement 


\section{Introduction}

Oral supplementation of membrane glycerolphospholipids provides patients with important cellular constituents that are damaged during chronic illnesses and aging [1]-[3]. These membrane phospholipids fulfill at least four major requirements for cell and tissue health [2] [3]. They are used as: 1) the matrix for all cellular membranes, enabling separation of enzymatic and chemical reactions into discrete cellular compartments; 2) an important energy storage reservoir; 3) bioactive molecules in certain signal transduction and molecular recognition pathways; and 4) important functional molecules that undergo interactions with other cellular constituents, such as proteins and glycoproteins that provide enzymatic function [3] [4].

Dietary sources often cannot provide enough essential lipids for maintenance of health, and in particular, for individuals with chronic illnesses [2] [3] [5]. Therefore, membrane glycerolphospholipids [1] [3] [6] or certain bioactive lipids [2] [5] at high concentrations have been used as supporting treatments or for health maintenance. Essential lipids, such as glycerolphospholipids, and fatty acids, are efficiently absorbed in the upper small intestine, almost all within hours of ingestion [3] [7] [8]. From there glycerolphospholipids and fatty acids are transported to every cell in a human to insure a source for the natural replacement of damaged membrane lipid components [3] [4] [9].

During aging (and in most if not all acute and chronic illnesses) cellular membranes are damaged by free radicals [3] [9]-[11]. When the production of reactive oxygen species (ROS), such as superoxide anion radicals, hydroxyl radicals and hydrogen peroxide, and reactive nitrogen species (RNS), such as peroxynitrite anion, are in excess of a cell's ability to neutralize these free radicals using natural antioxidants, damage to cellular structures occurs [3] [9]-[11]. Membrane lipids are especially sensitive to oxidative damage by ROS and RNS [3] [10] [11]. Thus oral supplementation of membrane lipids as membrane lipid replacement (MLR) molecules for cellular membranes and other structures, or as natural or synthetic bioactive lipids that can act as effector molecules in signaling and other cellular processes, can be used to restore cellular homostatasis [1] [3] [5] [9].

There is a rich history of using various lipid supplements to promote health. Readers are directed to various contributions, mainly reviews of the literature, for historical perspective [1]-[3] [5] [6] [9] [10].

\section{No Safety Issues}

There are no known safety issues with MLR. High doses of membrane phospholipids have been given to animals and humans with no apparent acute or chronic toxicity [3] [8] [12]. In phase I/II clinical trials on patients with cardiovascular disease, phosphatidylinositol was given at doses over $5 \mathrm{~g}$ per day with only positive effects. This membrane phospholipid was shown to increase plasma high-density lipoprotein cholesterol and apolipoprotein A1 levels and reduce triglyceride levels without any evidence of toxicity [13]. Long-term administration of relatively high oral doses of replacement membrane lipids has improved cardiovascular blood markers. For example, older subjects (average age $=60.7$ ) received over $2 \mathrm{~g}$ per day oral glycerolphospholipids $\left(\right.$ NTFactor ${ }^{\circledR}$, Table 1) for over 6 months and showed no evidence of adverse effects of the phospholipids. In fact, their cardiovascular blood marker levels, such as homocysteine, improved significantly during the trial $(p<0.001)$ [14]. Reviews of clinical studies on the use of MLR in the long-term treatment of cardiovascular and other diseases concluded that there was no evidence of any toxicity from glycerolphospholipids [3] [15].

The U.S. Federal Drug Administration (FDA) has classified membrane phospholipids used in MLR in the category of "Generally Recognized as Safe" (GRAS) [16].

\section{Membrane Lipid Replacement}

MLR supplements for membrane repair and replacement of damaged lipids have been developed that contain mixtures of the common membrane glycerolphospholipids, along with unsaturated fatty acids and other lipid components [2] [3] [6]. Of the commercially available oral MLR supplements, most are lecithin preparations that lack protection from oxidation and enzymatic destruction. There are oral MLR supplements, such as NTFactor ${ }^{\circledR}$ and NTFactor Lipids ${ }^{\circledR}$ (Table 1) that fulfill the requirements for convenience, efficacy, safety and protection against oxidation and degradation [1] [3]. They contain antioxidants and fructooligosaccharides to protect the glycerolphospholipids from oxidation, high or low temperature, and bile and enzymatic destruction [17]. These supplements contain suggested daily amounts of 1.8 to over $4 \mathrm{~g}$ of glycerolphospholipids with mostly unsaturated fatty acids (Table 1). Some NTFactor-containing supplements also contain probiotic bacteria, various 
Table 1. Lipid composition of NTFactor ${ }^{\text {बa }}$.

\begin{tabular}{ccc}
\hline Name & Abbreviation & Percent $[\mathrm{w} / \mathrm{w}]$ \\
Glycerolphospholipids & PC & 31.62 \\
Phosphatidylcholine & PI & 24.87 \\
Phosphatidylinositol & PE & 18.86 \\
Phosphatidylethanolamine & PA & 13.88 \\
Phosphatidic acid & DGDG & 5.88 \\
Digalactosyldiacylglycerol & PG & 2.37 \\
Phosphatidylglycerol & LPC & 0.98 \\
Lysophosphatidylcholine & LPE & 0.70 \\
Lysophosphatidyethanolamine & PS & 0.48 \\
Phosphatidylserine & MGDG & 0.31 \\
Monoglactosyldiacylglycerol & & 58.41 \\
Fatty Acids & $18: 2 \Delta 9,12(\mathrm{n}-6)$ & 19.39 \\
Linoleic acid & $16: 0$ & 9.68 \\
Palmitic acid & $18: 1 \Delta 9(\mathrm{n}-9)$ & 5.87 \\
Oleic acid & $18: 3 \Delta 9,12,15(\mathrm{n}-3)$ & 3.90 \\
Linolenic acid & $18: 0$ & \\
Stearic acid & &
\end{tabular}

${ }^{\mathrm{a}}$ NTFactor ${ }^{\circledR}$ is a patented product produced by Nutritional Therapeutics, Inc. of Hauppuage, NY.

vitamins and minerals and other ingredients. For example, a specific supplement for mitochondrial support, ATP Fuel $^{\circledR}$, contains 2 g per serving or 4 g per day of NTFactor ${ }^{\circledR}$ and also NADH, Coenzyme-Q10, vitamin E, alpha-ketoglutaric acid, L-carnitine, and other ingredients [18]. Other oral MLR products, such as those containing purified phosphatidylserine (PS), are also available [19].

An intravenous-delivered MLR mixture of glycerolphospholipids ("Essential phospholipids") can deliver high phospholipid concentrations without the need for inhibiting intestinal disruption [6]. However, this product is still susceptible to enzymatic and oxidative damage, and daily intravenous delivery comes with some risk for adverse events, such as infection, blood vessel damage, thrombosis, pruritus, dyspnoea, urticaria, among other potential problems.

\section{Clinical Uses of Membrane Lipid Replacement-Fatigue}

Until recently MLR was used primarily to treat fatigue [1] [3] [20]. Fatigue is the most common complaint of patients seeking general medical care [21] [22], and it is associated with most if not all chronic medical conditions as well as natural aging [21]. Fatigue is considered a complex, multidimensional sensation that is not well understood but is perceived to be a loss of overall energy, mental or physical tiredness, a feeling of exhaustion or diminished endurance, and an inability to perform even simple tasks without exertion [21]-[23]. At the cellular level moderate to severe fatigue has been related to loss of mitochondrial function and diminished production or leakage of ATP from mitochondria [20] [23] [24].

Fatigue develops during aging and chronic diseases due to a variety of additive and synergistic causes [20][25]. An important contributor to fatigue is oxidative damage to mitochondrial membrane lipids. This increases inner membrane leakiness and lowers trans-membrane potential of the inner mitochondrial membrane (MIM), thus impairing mitochondrial function and production of ATP, resulting in reduced physical and mental performance with aging and disease [20] [24]-[26]. For example, chronic fatigue syndrome patients present with evidence of oxidative damage to DNA and lipids [26] [27], such as oxidized blood markers and oxidized membrane lipids that are indicative of excess oxidative stress [27] [28]. These patients also have sustained, elevated levels of peroxynitrite due to excess nitric oxide, which can also result in lipid peroxidation and loss of mitochondrial function as well as changes in cytokine levels that exert a positive feedback on nitric oxide production [29]. 
MLR has been used in several clinical studies to treat moderate to severe chronic fatigue patients in order to reduce their fatigue levels [30]-[37] (Table 2). Although most of the clinical studies presented in Table 2 contained fewer than 50 patients and were open-label, there were significant outcomes in terms of fatigue reduction. For example, the effect of NTFactor ${ }^{\circledR}$ glycerolphospholipids on fatigue in moderately fatigued subjects was examined to see if mitochondrial function improved with oral administration of the MLR supplement [31]. In this cross-over clinical trial there was good correspondence between reductions in fatigue and gains in mitochondrial function. After 8 weeks of MLR with 3.9 g per day NTFactor ${ }^{\circledR}$, mitochondrial function was significantly improved, and after 12 weeks of NTFactor ${ }^{\circledR}$ supplementation, mitochondrial function was found to be similar to that found in young healthy adults $(26.8 \%$ increase, $\mathrm{p}<0.0001)$, while fatigue was reduced $35.5 \%(\mathrm{p}<0.001)$ [31]. After 12 weeks of supplement use, subjects were placed on placebo without their knowledge for an additional 12 weeks, and their fatigue and mitochondrial function were again measured. After the 12-week placebo period, fatigue and mitochondrial function were intermediate between the initial starting values and those found after eight or 12 weeks on the supplement [31]. Similar results on the effects of NTFactor ${ }^{\circledR}$ on fatigue were found in patients with chronic fatigue syndrome (CFS/ME) and/or fibromyalgia syndrome, Gulf War illness and chronic Lyme disease (reductions from 26\% - 43\%, Table 2). Taken together, these clinical data indicate that MLR can be successfully employed to reduce fatigue in different chronic conditions.

Loss of mitochondrial function is not always related exclusively to membrane lipid damage [20]. Thus supplements containing Coenzyme Q10, L-carnitine, alpha-lipoic acid, NADH along with membrane glycerolphospholipids have has also been used in combination mitochondrial supplement studies (for example, ATP Fuel ${ }^{\circledR}$ ) to treat long-term chronic illness patients with intractable fatigue [36] [37]. The patients in these clinical studies had been ill with moderate to severe intractable fatigue for an average of over 17 years, had been seen by many physicians (>15), and had taken an average of over 35 supplements or drugs with no effect on their fatigue [36] [37]. Although the numbers of patients enrolled in the studies were limited, they responded with significant reductions in fatigue within 60 days $(\mathrm{p}<0.0001)$. Regression analysis of the data indicated that the reductions in fatigue were gradual, consistent, and occurred with a high degree of confidence $\left(R^{2}=0.960-0.998\right)$. The combination supplement proved to be a safe and effective method to significantly reduce fatigue in patients with long-term intractable fatigue [36] [37].

In addition to fatigue in chronic illnesses, fatigue is also one of the most common symptoms of cancer. It occurs in cancer patients from the earliest forms of cancer to the most progressed forms of metastatic disease [35]. Cancer-associated fatigue is not well understood, but it is thought to be due to a combination of the effects of cancer itself plus the effects of cancer treatments [35] [38] [39]. MLR has been used to treat cancer-associated fatigue and the fatigue-effects of cancer therapy [35] [40]. For example, NTFactor ${ }^{\circledR}$ in a vitamin-mineral formulation (Propax ${ }^{\mathrm{TM}}$ ) was used to reduce some of the adverse effects of cancer therapy, such as chemotherapy-induced fatigue, nausea, vomiting, malaise, diarrhea, headaches and other side effects [39]. In advanced metastatic colon, pancreatic and rectal cancer patients this supplement reduced the adverse effects of chemotherapy, resulting in significantly fewer episodes of fatigue, nausea, diarrhea, constipation, skin changes, insomnia and

Table 2. Some clinical effects of dietary MLR supplement NTFactor ${ }^{\circledR}$ on fatigue scores. ${ }^{a}$

\begin{tabular}{|c|c|c|c|c|c|}
\hline Subjects/patients & $\mathrm{n}$ & Av age on MLR & Time on MLR & Fatigue scale reduction (\%) & Reference \\
\hline Chronic fatigue $^{c}$ & 34 & 50.3 & 8 wks & $40.5^{* *}$ & Ellithorpe et al. [30] \\
\hline Aging, chronic fatigue $^{\mathrm{d}}$ & 22 & 68.9 & 12 wks & $35.5^{*}$ & Agadjanyan et al. [31] \\
\hline Chronic fatigue syndrome $^{\mathrm{d}}$ & 15 & 44.8 & 8 wks & $43.1^{*}$ & Nicolson \& Ellithorpe [32 \\
\hline Obesity, fatigue $^{e}$ & 35 & 42 & 8 wks & $23.9 *$ & Ellithrope et al. [33] \\
\hline Aging, chronic fatigue ${ }^{\mathrm{f}}$ & 67 & 57.3 & $1 \mathrm{wk}$ & $36.8^{* *}$ & Nicolson et al. [34] \\
\hline Cancer, fatigue $^{c}$ & 35 & 50.7 & 8 wks & $30.1^{*}$ & Nicolson [35] \\
\hline Chronic fatigue syndrome $\mathrm{e}^{\mathrm{g}, \mathrm{h}}$ & 30 & 55.0 & 8 wks & $30.7^{* *}$ & Nicolson et al. [36] \\
\hline Lyme disease, fatigue $^{\mathrm{g}, \mathrm{h}}$ & 17 & 52.4 & 8 wks & $26^{*}$ & Nicolson et al. [37] \\
\hline Gulf War Illness, fatigue ${ }^{\mathrm{g}, \mathrm{h}}$ & 16 & 42.2 & 8 wks & $34.6^{*}$ & Nicolson et al. [36] \\
\hline
\end{tabular}

${ }^{\mathrm{a}}$ Modified from Nicolson and Ash [3], ${ }^{\mathrm{b}}$ Piper Fatigue Scale [38], ${ }^{\mathrm{C}}$ Propax ${ }^{\mathrm{TM}}$ with NTFactor ${ }^{\circledR}$, ${ }^{\mathrm{d}}$ NTFactor ${ }^{\circledR}$, ${ }^{\mathrm{e}}$ Healthy Curb $^{\mathrm{TM}}$ with NTFactor ${ }^{\circledR},{ }^{\mathrm{f}}$ Advanced Physician's Formula ${ }^{\mathrm{TM}}$ with NTFactor ${ }^{\circledR},{ }^{\mathrm{g}}$ ATP Fuel ${ }^{\circledR}$ with NTFactor ${ }^{\circledR}$, ${ }^{\mathrm{h}}$ Intractable fatigue ( $>17.1$ years), ${ }^{* *} \mathrm{p}<0.0001$, ${ }^{*} \mathrm{p}<0.001$ compared to without NTFactor determined by t-test ${ }^{\circledR}$. 
other effects as assessed by nurses and separately by the patients themselves. Eighty-one percent of the patients on chemotherapy that used Propax ${ }^{\mathrm{TM}}$ experienced an overall improvement in quality of life parameters. In a subsequent double-blind, placebo-controlled cross-over trial 36 patients on chemotherapy plus Propax ${ }^{\mathrm{TM}}$ showed fewer adverse effects, resulting in improvements in fatigue, nausea, diarrhea, impaired taste, constipation, insomnia and other quality of life indicators [40].

\section{Other Clinical Uses of Membrane Lipid Replacement}

In other clinical studies, MLR may modify metabolism through body mass reduction and appetite restraint [33]. In this study 30 normal obese subjects used oral HealthyCurb ${ }^{\circledR}$, an NTFactor ${ }^{\circledR}$ and alpha-amylase inhibitor formulation, 30 min before each meal. Subjects gradually lost weight, with significant reductions in waist and hip circumferences, along with reductions in body mass index (BMI) and basal metabolic rate (BMR) $(\mathrm{p}<0.001)$. Overall hunger was reduced $44.5 \%(\mathrm{p}<0.001)$, with reduced cravings for sweets and fats, and there was a $23.9 \%$ reduction in fatigue $(\mathrm{p}<0.009)$. Along with fatigue reduction there was a $26.8 \%$ perceived improvement in cognition and ability to concentrate, remember and think clearly ( $<<0.004)$ [33].

Mental clarity has been examined in a preliminary study using NTFactor Lipids ${ }^{\circledR}$ [41]. A group of 29 subjects were given $0.6 \mathrm{~g}$ of NTFactor Lipids ${ }^{\circledR}$ in a drink, and fatigue and mental focus were surveyed after three hours. At this time a self-reported survey instrument was given to the participants. A majority of subjects responded within one hour, and by three hours they reported perceived improvements in cognition function, mental clarity and focus along with fatigue reductions [41].

Specific glycerolphospholipids, such as oral phosphatidylserine (PS), have been used in clinical studies to assess improvements in memory loss and cognition. In 30 male and female subjects (aged 50 - 90 years old, average 74.6 years old) with memory impairments unrelated to neurological disease, stroke, diabetes, infections or inflammatory processes, a 12-week study was initiated to determine if $300 \mathrm{mg}$ PS per day modified outcomes in 6 different tests of memory and cognition [42]. At the end of the trial participants showed significant improvements in memory recognition ( $p=0.004)$ and recall $(p=0.006)$, total learning $(p=0.013)$, executive functions $(\mathrm{p}=0.004)$, metal flexibility, and visual spatial learning. There were no adverse events during the trial, and interestingly both mean systolic and diastolic blood pressure values were reduced at 12 weeks in comparison to baseline values [42]. Kato-Kataoka et al. [43] conducted a similar double-blind, randomized study on 78 subjects (50 - 69 years old) to determine if 100 - $300 \mathrm{mg}$ oral PS per day versus placebo affected memory scores. They found that PS significantly improved behavioral memory functions, especially short-term memory and cognitive function in low-scoring (delayed word recall) participants $(\mathrm{p}<0.01)$ [43].

Oral MLR preparations have been used to treat memory loss in aged subjects and in Alzheimer's disease (AD). AD patients supplemented with $300 \mathrm{mg}$ per day bovine PS for 6 months showed cognitive improvement relative to placebo controls [44]; however, this was not seen in another study on elderly subjects with age-associated memory impairment that received 300 - $600 \mathrm{mg}$ soy PS daily for 12 weeks [19]. In these and other studies the PS was found to be completely safe [45].

\section{Membrane Lipid Replacement-Studies on Mitochondrial Function}

Since mitochondrial function is related to symptoms like fatigue, it is appropriate to ask whether this is a silent relationship. In fact, fatigue in general, and fatiguing illnesses in particular, are characterized by losses in mitochondrial function [20] [23] [24] [46] [47]. Filler et al. [23] have reviewed the literature on fatigue and the association of mitochondrial dysfunction and its possible causes. In addition, they examined the association of the levels of various mitochondrial metabolites, such as acylcarnitine and coenzyme Q10, with fatigue in selected publications [23].

Although some clinical conditions associated with fatigue are clearly related to mitochondrial dysfunction, such as chronic fatigue syndrome (CFS/ME), fibromyalgia syndrome, cancer, among other conditions, others are less clearly associated with loss of mitochondrial function. Various mental disorders, such as schizophrenia, bipolar disease, and others, may be associated with mitochondrial dysfunction, but the links are less clear [23]. These patients are also often characterized with moderate to severe depression. Depression is a commonly found comorbidity in cancer, mental disorders and other conditions, where it shows physical, cognitive and emotional overlap with fatigue in individual patients [48].

Various mitochondrial supplements have been used to treat mitochondrial dysfunction and alleviate or more 
commonly reduce fatigue [1] [3] [20] [23] [39] [49]. However, in only a few studies were the losses of mitochondrial function directly related to fatigue scores. A cross-over trial discussed in the previous section directly related loses or gains in mitochondrial function in individual patients to their fatigue scores [31]. In this case the oral use of NTFactor ${ }^{\circledR}$ glycerolphospholipids resulted in significant improvements in mitochondrial function in patients with various diagnoses plus chronic fatigue [31]. I have listed the studies that have used MLR, specifically NTFactor ${ }^{\circledR}$, to reduce fatigue in Table 2.

MLR can also have negative consequences, if the wrong membrane lipids are used. For example, feeding rodents a rapeseed oil-rich diet was found to alter hepatic mitochondrial membrane lipid composition and reduce mitochondrial function [50]. In this animal study hepatic mitochondria were examined after rats were fed the rapeseed oil diet for 22 days, after which they were found to express differences in the relative proportions of glycerolphospholipid classes (increase in PC to PE ratio and differences in cardiolipins) and ratios of saturated to unsaturated fatty acids in their mitochondrial membranes. These mitochondrial membrane lipid alterations paralleled the negative changes in mitochondrial energetics [50].

\section{Membrane Lipid Replacement-In Vitro Studies}

There has been a tendency in recent years to reduce the complexity of living systems down to studying cell organelles and even single molecules [51]. In this regard experiments designed to test the role of certain phospholipids on function have utilized isolated organelles, such as mitochondria, and to these isolates have been added specific glycerolphospholipids to test the effects of MLR. For example, Shaikh et al. [52] prepared small PC vesicles or PC/cardiolipin vesicles and incubated these with isolated, suspended rat heart mitochondria in vitro. The vesicles were observed to fuse with the mitochondria, resulting in reductions in electron transport enzymatic activities. Thus the authors concluded that increasing mitochondrial membrane phospholipid content lowers electron transport enzymatic activities [52]. There are a number of problems with this approach, including the misinterpretation that this in vitro experiment is the same as MLR. The authors used only one glycerolphospholipid (PC) with one fatty acid (oleic) with or without cardiolipin at non-physiological concentrations, and they did not account for dilution of the mitochondrial membrane components in order to incorporate the excess phospholipids [52].

MLR is the natural in vivo replacement of damaged membrane phospholipids, not the in vitro excess packing of phospholipids into isolated organelles or organelle membranes. In the case of mitochondria, the relative amounts and ratios of membrane phospholipids-to-electron transport complexes are important for functional activity [53]. Mitochondrial membranes also contain more than just PC and cardiolipin, and in the case of the mitochondrial inner membrane, phosphotidylethanolamine (PE) is functionally important in the stabilities, interactions and activities of membrane respiratory chain complexes [54]. PE depletion results in decreased transmembrane potential across the inner mitochondrial membrane, and this results in impaired mitochondrial function [54]. In addition, PE has been shown to be important in regulating membrane fluidity [55]. Fusion of phospholipid vesicles with organelles like mitochondria results in organelle swelling and dilution of membrane components that are normally compacted into dense super complex structures for maximal interactions [56].

A similar in vitro phenonmenon was seen when intact spermatozoa were incubated with glycerolphospholipid vesicles generated from NTFactor Lipids ${ }^{\circledR}$ [57]. At lower glycerolphospholipid concentrations vesicle fusion with sperm resulted in enhanced motility [57], which is directly related to mitochondrial activity [58]. However, this was not seen at higher concentrations of the vesicles where membrane swelling due to incorporation of excess membrane glycerolphospholipids occurred [57]. Thus it is important to maintain the compact nature of cellular membranes to retain biological activity [58]-[60]. The fusion of high, nonphysiological concentrations of glycerolphospholipids into mitochondrial membranes in vitro cannot be expected to mimic natural in vivo MLR.

\section{Conclusions}

Using oral MLR supplements in clinical trials has shown that MLR is a safe and effective method to replace damaged membrane glycerolphospholipids in order to restore membrane function. Replacing damaged membrane phospholipids has a positive effect on cellular functions, such as mitochondrial function, but MLR is not limited to a single organelle. The positive effects of MLR also occur in other membrane compartments, such as the plasma membrane, and future studies will undoubtedly concentrate on the functional effects of MLR on the 
plasma membranes of epithelial cells, nerve cells, and other cells as well as on various intracellular membranes. Since MLR natural supplements are not drugs, the usual drug safety issues are not applicable. Extremely high doses of MLR glycerolphospholipids have been given to animals and humans over long periods of time without any toxic or adverse effects [3] [16]. Thus MLR glycerolphospholipids are extremely safe to use as a daily dietary supplement or for treatment of specific membrane deficits, such as loss of mitochondrial MIM function.

It should be clear from this contribution that additional clinical studies are needed to further examine the role of MLR supplements in enhancing mitochondrial function in vivo and reducing symptoms like fatigue. With two exceptions [31] [40], the MLR studies reviewed in this contribution were open label, not blinded, placebo-controlled studies. In the cross-over trial by Agadjanyan et al. [31], a variety of patients with various diagnoses that had moderate to severe chronic fatigue were studied. The trial could have been improved if patients were enrolled that presented with one primary diagnosis where fatigue was a major symptom, such as chronic fatigue syndrome. Also, with one exception [34], the clinical studies in Table 2 utilized less than 50 patients. Thus, more robust, blinded, placebo-controlled studies with more uniform cohorts of patients would be an improvement for studying the relationship between fatigue, mitochondrial function and the effects of MLR supplements.

The potential uses of MLR supplements to improve health and treat various clinical conditions are numerous and widespread [2] [3] [5] [61]. In fact, this review has presented some limited data on the potential uses of MLR supplements to improve mitochondrial function, but the future uses of MLR are much broader than mitochondria and are extended to many aspects of membrane health and function. New formulations of MLR supplements directed primarily at restoring damage in plasma membranes, nuclear membranes and other intracellular membranes will likely be important in future studies.

\section{Acknowledgements}

This contribution was supported by grants and contracts to the Institute for Molecular Medicine, a non-profit, tax-exempt research organization under section 501 (c) (3) of the U.S. tax code. Professor emeritus Garth Nicolson is a part-time consultant to the following: Nutritional Therapeutics, Inc. of Hauppuage, NY; Resarched Nutritionals of Los Olivos, CA and Naturally Plus USA of Las Vegas, NV.

\section{References}

[1] Nicolson, G.L. (2003) Lipid Replacement as an Adjunct Therapy in Chronic Fatigue, Anti-Aging and Restoration of Mitochondrial Function. Journal of the American Nutraceutical Association, 6, 22-28. http://www.immed.org/treatment\%20considerations/GNicolsonJANAvol6_3_.pdf

[2] Küllenberg, D., Taylor, L.A., Schneider, M. and Massing, U. (2012) Health Effects of Dietary Phospholipids. Lipids in Health and Disease, 11, e3. http://dx.doi.org/10.1186/1476-511X-11-3

[3] Nicolson, G.L. and Ash, M.E. (2014) Lipid Replacement Therapy: A Natural Medicine Approach to Replacing Damaged Lipids in Cellular Membranes and Organelles and Restoring Function. Biochimica et Biophysica Acta (BBA)Biomembranes, 1838, 1657-1679. http://dx.doi.org/10.1016/j.bbamem.2013.11.010

[4] van Meer, G., Voelker, D.B. and Feigenson, G.W. (2008) Membrane Lipids: Where They Are and How They Behave. Nature Reviews Molecular and Cell Biology, 9, 112-124. http://dx.doi.org/10.1038/nrm2330

[5] Escribá, P.V. (2006) Membrane-Lipid Therapy: A New Approach in Molecular Medicine. Trends in Molecular Medicine, 12, 34-43. http://dx.doi.org/10.1016/j.molmed.2005.11.004

[6] Gundermann, K.J., Kuenker, A., Kuntz, E. and Droździk, M. (2011) Activity of Essential Phospholipids (EPL) from Soybean in Liver Diseases. Pharmacology Reports, 63, 643-659. http://dx.doi.org/10.1016/S1734-1140(11)70576-X

[7] Le Kim, D. and Betzing, H. (1976) Intestinal Absorption of Polyunsaturated Phosphatidylcholine in the Rat. Hoppe Seylers Zeitschriftfür Physiologische Chemie, 357, 1321-1331. http://dx.doi.org/10.1515/bchm2.1976.357.2.1321

[8] Zierenberg, O. and Grundy, S.M. (1982) Intestinal Absorption of Polyenephosphatidylcholine in Man. Journal of Lipid Research, 23, 1136-1142.

[9] Ibarguren, M., López, D.J. and Escribá, P.V. (2014) The Effect of Natural and Synthetic Fatty Acids on Membrane Structure, Microdomain Organization, Cellular Functions and Human Health. Biochimica et Biophysica Acta (BBA)Biomembranes, 1838, 1518-1528. http://dx.doi.org/10.1016/j.bbamem.2013.12.021

[10] Adibhatla, R.M. and Hatcher, J.F. (2010) Lipid Oxidation and Peroxidation in CNS Health and Disease: From Molecular Mechanisms to Therapeutic Opportunities. Antioxidants and Redox Signaling, 12, 125-169.

http://dx.doi.org/10.1089/ars.2009.2668 
[11] Catalá, A. (2012) Lipid Peroxidation Modifies the Picture of Membranes from the "Fluid Mosaic Model” to the "Lipid Whisker Model”. Biochimie, 94, 101-109. http://dx.doi.org/10.1016/j.biochi.2011.09.025

[12] Wagener, H.H., Fontaine, R. and Neumann, B. (1976) Pharmakologie "Essentiele" Phospholipide (EPL). Drug Research, 26, 1733-1743.

[13] Pandey, N.R. and Sparks, D.L. (2008) Phospholipids as Cardiovascular Therapeutics. Current Opinion in Investigative Drugs, 9, 281-285.

[14] Ellithorpe, R.R., Settineri, R., Ellithorpe, T. and Nicolson, G.L. (2015) Blood Homocysteine and Fasting Insulin Levels Are Reduced and Erythrocyte Sedimentation Rates Increased with a Glycophospholipid-Vitamin Formulation: A Retrospective Study in Older Subjects. Functional Foods in Health and Disease, 5, 126-135. http://functionalfoodscenter.net/files/102987419.pdf

[15] Cohn, J.S., Wat, E., Kamili, A. and Tandy, S. (2008) Dietary Phospholipids, Hepatic Metabolism and Cardiovascular Disease. Current Opinion in Lipidology, 19, 257-262. http://dx.doi.org/10.1097/MOL.0b013e3282ffaf96

[16] US Federal Drug Administration (1970) Scientific Literature Reviews on Generally Recognized as Safe (GRAS) Food Ingredients: Lecithins. GRAS Report, PB-241 970.

[17] Hendry, G.A.F. (1993) Evolutionary Origins and Natural Functions of Fructans-A Climatological, Biogeographic and Mechanistic Appraisal. New Phytology, 123, 3-14. http://dx.doi.org/10.1111/j.1469-8137.1993.tb04525.x

[18] Nicolson, G.L., Settineri, R. and Ellithorpe, R. (2012) Lipid Replacement Therapy with a Glycophospholipid Formulation with NADH and CoQ10 Significantly Reduces Fatigue in Intractable Chronic Fatiguing Illnesses and Chronic Lyme Disease. International Journal of Clinical Medicine, 3, 163-170. http://dx.doi.org/10.4236/ijcm.2012.33034

[19] Jorissen, B.L., Brouns, F., van Boxtel, M.P., Ponds, R.W., Verhey, F.R., Jolles, J. and Riedel, W.J. (2001) The Influence of Soy-Derived Phosphatidylserine on Cognition in Age-Associated Memory Impairment. Nutrition and Neuroscience, 4, 121-134.

[20] Nicolson, G.L. (2014) Mitochondrial Dysfunction and Chronic Disease: Treatment with Natural Supplements. Alternative Therapies in Health and Medicine, 20, 18-25.

http://www.immed.org/treatmentconsiderations/05.23.14.TreatmentConsiderations/Mito_Dysfunct_Treatm-NicolsonA THD2014.pdf

[21] Kroenke, K., Wood, D.R., Mangelsdorff, A.D., Meier, N.J. and Powell, J.B. (1988) Chronic Fatigue in Primary Care. Prevalence, Patient Characteristics, and Outcome. Journal of the American Medical Association, 260, 929-934. http://dx.doi.org/10.1001/jama.1988.03410070057028

[22] Morrison, J.D. (1980) Fatigue as a Presenting Complaint in Family Practice. Journal of Family Practice, 10, $795-801$.

[23] Filler, K., Lyon, D., Bennett, J., McCain, N., Elswick, R., Lukkahatai, N. and Saligan, L.N. (2014) Association of Mitochondrial Dysfunction and Fatigue: A Review of the Literature. BBA Clinical, 1, 12-23. http://dx.doi.org/10.1016/j.bbacli.2014.04.001

[24] Myhill, S., Booth, N.E. and McLaren-Howard, J. (2009) Chronic Fatigue Syndrome and Mitochondrial Dysfunction. International Journal of Clinical and Experimental Medicine, 2, 1-16.

[25] Krupp, L.B. and Pollina, D.A. (1996) Mechanisms and Management of Fatigue in Progressive Neurological Disorders. Current Opinion in Neurology, 9, 456-460. http://dx.doi.org/10.1097/00019052-199612000-00011

[26] Manuel y Keenoy, B., Moorkens, G., Vertommen, J. and De Leeuw, I. (2001) Antioxidant Status and Lipoprotein Peroxidation in Chronic Fatigue Syndrome. Life Sciences, 68, 2037-2049. http://dx.doi.org/10.1016/S0024-3205(01)01001-3

[27] Fulle, S., Mecocci, P., Fano, G., Vecchiet, I., Racciotti, D., Cherubini, A., Pizzigallo, E., Vecchiet, L., Senin, U. and Beal, M.F. (2000) Specific Oxidative Alterations in Vastus Lateralis Muscle of Patients with the Diagnosis of Chronic Fatigue Syndrome. Free Radical Biology and Medicine, 29, 1252-1259. http://dx.doi.org/10.1016/S0891-5849(00)00419-6

[28] Richards, R.S., Roberts, T.K., McGregor, N.R., Dunstan, R.H. and Butt, H.L. (2000) Blood Parameters Indicative of Oxidative Stress Are Associated with Symptom Expression in Chronic Fatigue Syndrome. Redox Reports, 5, 35-41. http://dx.doi.org/10.1179/rer.2000.5.1.35

[29] Pall, M.L. (2000) Elevated, Sustained Peroxynitrite Levels as the Cause of Chronic Fatigue Syndrome. Medical Hypotheses, 54, 115-125. http://dx.doi.org/10.1054/mehy.1998.0825

[30] Ellithorpe, R.R., Settineri, R. and Nicolson, G.L. (2003) Reduction of Fatigue by Use of a Dietary Supplement Containing Glycophospholipids. Journal of the American Nutraceutical Association, 6, 23-28.

[31] Agadjanyan, M., Vasilevko, V., Ghochikyan, A., Berns, P., Kesslak, P., Settineri, R. and Nicolson, G.L. (2003) Nutritional Supplement (NTFactor) Restores Mitochondrial Function and Reduces Moderately Severe Fatigue in Aged Subjects. Journal of Chronic Fatigue Syndrome, 11, 23-36. http://dx.doi.org/10.1300/J092v11n03 03 http://www.immed.org/treatment\%20considerations/06.16.12/AgadjanyanNicolsonJCFS.pdf 
[32] Nicolson, G.L. and Ellithorpe, R. (2006) Lipid Replacement and Antioxidant Nutritional Therapy for Restoring Mitochondrial Function and Reducing Fatigue in Chronic Fatigue Syndrome and Other Fatiguing Illnesses. Journal of Chronic Fatigue Syndrome, 13, 57-68. http://dx.doi.org/10.1300/J092v13n01_06 http://www.immed.org/publications/Nicolson_ElllithorpeJCFS_copy.pdf

[33] Ellithorpe, R.A., Settineri, R., Jacques, B. and Nicolson, G.L. (2012) Lipid Replacement Therapy Functional Food with NTFactor for Reducing Weight, Girth, Body Mass, Appetite, Cravings for Foods and Fatigue While Improving Blood Lipid Profiles. Functional Foods in Health and Disease, 2, 11-24. http://ffhdj.com/index.php/ffhd/article/view/102/212

[34] Nicolson, G.L., Ellithorpe, R.R., Ayson-Mitchell, C., Jacques, B. and Settineri, R. (2010) Lipid Replacement Therapy with a Glycophospholipid-Antioxidant-Vitamin Formulation Significantly Reduces Fatigue within One Week. Journal of the American Nutraceutical Association, 13, 11-15. http://www.ana-jana.org/reprints/JANA13-1Reprint-GarthNicolson.pdf

[35] Nicolson, G.L. (2010) Lipid Replacement Therapy: A Nutraceutical Approach for Reducing Cancer-Associated Fatigue and the Adverse Effects of Cancer Therapy While Restoring Mitochondrial Function. Cancer Metastasis Reviews, 29, 543-552. http://dx.doi.org/10.1007/s10555-010-9245-0

[36] Nicolson, G.L., Settineri, R. and Ellithorpe, R.R. (2012) Lipid Replacement Therapy with a Glycophospholipid Formulation with NADH and CoQ10 Significantly Reduces Fatigue in Intractable Chronic Fatiguing Illnesses and Chronic Lyme Disease. International Journal of Clinical Medicine, 3, 163-170. http://dx.doi.org/10.4236/ijcm.2012.33034

[37] Nicolson, G.L., Settineri, R. and Ellithorpe, R.R. (2012) Glycophospholipid Formulation with NADH and CoQ10 Significantly Reduces Intractable Fatigue in Western Blot-Positive Chronic Lyme Disease Patients: Preliminary Report. Functional Foods in Health and Disease, 2, 35-47. http://ffhdj.com/index.php/ffhd/article/view/100/208

[38] Piper, B.F., Linsey, A.M. and Dodd, M.J. (1987) Fatigue Mechanism in Cancer. Oncology Nursing Forum, 14, 17-23.

[39] Nicolson, G.L. and Conklin, K.A. (2008) Reversing Mitochondrial Dysfunction, Fatigue and the Adverse Effects of Chemotherapy of Metastatic Disease by Molecular Replacement Therapy. Clinical and Experimental Metastasis, 25, 161-169. http://dx.doi.org/10.1007/s10585-007-9129-z

[40] Colodny, L., Lynch, K., Farber, C., Papish, R., Phillips, K., Sanchez, M., Cooper, K., Pickus, O., Palmer, D., Percy, T.B., Faroqui, M. and Block, J.B. (2001) Results of a Study to Evaluate the Use of Propax to Reduce Adverse Effects of Chemotherapy. Journal of the American Nutraceutical Association, 3, 17-25.

[41] Ellithorpe, R.R., Settineri, R., Mitchell, C.A., Jacques, B., Ellithorpe, T. and Nicolson, G.L. (2011) Lipid Replacement Therapy Drink Containing a Glycophospholipid Formulation Rapidly and Significantly Reduces Fatigue While Improving Energy and Mental Clarity. Functional Foods in Health and Disease, 1, 245-254. http://ffhdj.com/index.php/ffhd/article/view/126/259

[42] Richter, Y., Herzog, Y., Lifshitz, Y., Hayun, R. and Zchut, S. (2103) The Effect of Soybean Phosphatidylserine on Cognitive Performance in Elderly with Subjective Memory Complaints: A Pilot Study. Clinical Interventions in Aging, 8, 557-563.

[43] Kato-Kataoka, A., Sukai, M., Ebina, R., Nonaka, C., Asano, T. and Miyamori, T. (2010) Soybean-Derived Phosphatidylserine Improves Memory Function of Elderly Japanese Subjects with Memory Complaints. Journal of Clinical Biochemistry and Nutrition, 47, 246-255. http://dx.doi.org/10.3164/jcbn.10-62

[44] Cernacchi, T., Bertoldin, T., Farina, C., Flori, M.G. and Crepaldi, G. (1993) Cognitive Decline in the Elderly: A Double-Blind, Placebo-Controlled Multicenter Study on the Efficacy of Phosphatidylserine Administration. Aging (Milano), 5, 123-133.

[45] Jorissen, B.L., Brouns, F., Van Baxtel, M.P. and Riedel, W.J. (2002) Safety of Soy-Derived Phosphatidylserine in Elderly People. Nutrition and Neuroscience, 5, 337-343. http://dx.doi.org/10.1080/1028415021000033802

[46] Booth, N.E., Mayhill, S. and McLaren-Howard, J. (2012) Mitochondrial Dysfunction and the Pathophysiology of Myalgic Encephalomyelitis/Chronic Fatigue Syndrome (ME/CFS). International Journal of Clinical and Experimental Medicine, 5, 208-220.

[47] Gorman, G.S., Elson, J.L., Newman, J., Payne, B., McFarland, R., Newton, J.L. and Turnbull, D.M. (2015) Perceived Fatigue Is Highly Prevalent and Debilitating in Patients with Mitochondrial Disease. Neuromuscular Disorders, 25, 563-566. http://dx.doi.org/10.1016/j.nmd.2015.03.001

[48] Brown, L.F. and Kroenke, K. (2009) Cancer-Associated Fatigue and Its Association with Depression and Anxiety: A Systematic Review. Psychosomatics, 50, 440-447. http://dx.doi.org/10.1016/S0033-3182(09)70835-7

[49] Myhill, S., Booth, N.E. and McLaren-Howard, J. (2013) Targeting Mitochondrial Dysfunction in the Treatment of Myalgic Encephalomyelitis/Chronic Fatigue Syndrome (ME/CFS)—A Clinical Audit. International Journal of Clinical and Experimental Medicine, 6, 1-15.

[50] Monteiro, J.P., Pereira, C.V., Silva, A.M., Maciel, E., Baldeiras, I., Peixoto, F., Domingues, M.R., Jurado, A.S. and Oliveira, P.J. (2013) Rapeseed Oil-Rich Diet Alters Hepatic Mitochondrial Membrane Lipid Composition and Disrupts Bioenergetics. Archives of Toxicology, 87, 2151-2163. http://dx.doi.org/10.1007/s00204-013-1068-7 
[51] Duzdevich, D. and Greene, E.C. (2012) Towards Physiological Complexity with in Vitro Single-Molecule Biophysics. Philosophical Transactions of the Royal Society London B: Biological Sciences, 368, Article ID: 20120271. http://dx.doi.org/10.1098/rstb.2012.0271

[52] Shaikh, S.R., Sullivan, E.M., Alleman, R.J., Brown, D.A. and Zeczycki, T.N. (2014) Increasing Mitochondrial Membrane Phospholipid Content Lowers the Enzymatic Activity of Electron Transport Complexes. Biochemistry, 53, 55895591. http://dx.doi.org/10.1021/bi500868g

[53] Ahang, M., Mileykovskaya, E. and Dowhan, W. (2002) Gluing the Respiratory Chains Together. Cardiolipin Is Required for Supercomplex Formation in the Inner Mitochondrial Membrane. Journal of Biological Chemistry, 277, $43533-43556$.

[54] Böttinger, L., Horvath, S.E., Kleinschroth, T., Hunte, C., Daum, G., Pfanner, N. and Becker, T. (2012) Phosphatidylethanolamine and Cardiolipin Differentially Affect the Stability of Mitochondrial Respiratory Chain Supercomplexes. Journal of Molecular Biology, 248, 677-686. http://dx.doi.org/10.1016/j.jmb.2012.09.001

[55] Dawaliby, R., Trubbia, C., Delporte, C., Noyon, C., Ruysschaert, J.-M., Van Antwerpen, P. and Guvaerts, C. (2015) Phosphatidylethanolamine Is a Key Regulator of Membrane Fluidity in Eukaryotic Cells. Journal of Biological Chemistry, 291, 3658-3667. http://dx.doi.org/10.1074/jbc.M115.706523

[56] Mileykovskaya, E., Penczek, P.A., Fang, J., Mallampalli, V.K., Sparagna, G.C. and Dowhan, W. (2012) Arrangement of the Respiratory Chain Complexes in Saccharomyces cerevisiae Supercomplex $\mathrm{III}_{2} \mathrm{IV}_{2}$ Revealed by Single Particle Cryo-Electron Microscopy. Journal of Biological Chemistry, 287, 23095-23103. http://dx.doi.org/10.1074/jbc.M112.367888

[57] Costa, C., Gonzales, T., Ferreira, G. and Nicolson, G.L. (2015) Lipid Replacement with a Membrane Glycerol Phospholipid Formulation: Enhancement of Spermatoozoa Motility and Viability. In: Martirosyan, D., Welty, F. and Zhou, J.-R., Eds., Functional and Medical Foods for Chronic Diseases: Bioactive Compounds and Biomarkers, Volume 18, Food Science Publisher, Dallas, 202-206.

[58] Plaza Davila, M., Martin Muñoz, P., Tapla, J.A., Ortega Ferrusola, C., Balao da Silva, C.C. and Peña, F.J. (2015) Inhibition of Mitochondrial Complex I Leads to Decreased Motility and Membrane Integrity Related to Increased Hydrogen Peroxide and Reduced ATP Production, While the Inhibition of Glycolysis Has Less Impact on Sperm Motility. PLOS ONE, 10, e0138777. http://dx.doi.org/10.1371/journal.pone.0138777

[59] Nicolson, G.L. (2014) The Fluid-Mosaic Model of Membrane Structure: Still Relevant to Understanding the Structure, Function and Dynamics of Biological Membranes after More Than 40 Years. Biochimica et Biophysica Acta (BBA)—Biomembranes, 1838, 1451-1466. http://dx.doi.org/10.1016/j.bbamem.2013.10.019

[60] Goñi, F.M. (2014) The Basic Structure and Dynamics of Cell Membranes: An Update of the Singer-Nicolson Model. Biochimica et Biophysica Acta, 1838, 1467-1476. http://dx.doi.org/10.1016/j.bbamem.2014.01.006

[61] Nicolson, G.L., Rosenblatt, S., Ferreira de Mattos, G., Settineri, R., Breeding, P.C., Ellithorpe, R.R. and Ash, M.E. (2016) Clinical Uses of Membrane Lipid Replacement Supplements in Restoring Membrane Function and Reducing Fatigue in Chronic Diseases and Cancer. Discoveries, 4, e54. 


\section{Abbreviations}

ATP: adenosinetriphosphate;

CFS: chronic fatigue syndrome;

CL: cardiolipin;

DHA: docosahexaenoic acid;

EPA: eicosapentaenoic acid;

ETC: electron transport chain;

FA: fatty acid;

FDA: US Federal Drug Administration;

GRAS: generally recognized as safe;

MDA: malondialdehyde;

ME: myalgic encephalomyelitis;

MIM: mitochondrial inner membrane;

MLR: membrane lipid replacement;

PC: phosphatidylcholine;

PE: phosphatidylethanolamine;

PG: phosphatidylglycerol;

PI: phosphatidylinositol;

PS: phosphatidylserine;

RNS: reactive nitrogen species;

ROS: reactive oxygen species. 